DOI $10.31489 / 2020$ No2/138-145

UDC 616.874

\title{
ANALYSIS OF THE APPLICABILITY OF PHYSICAL MODELS TO DESCRIBE DENSIFICATION OF LITHIUM FERRITE COMPACTS DURING SINTERING IN THE FIELD OF INTENSE ELECTRON BEAM
}

\author{
Surzhikov A.P. ${ }^{1}$, Lysenko E.N. ${ }^{1}$, Malyshev A.V. ${ }^{1}$, Kassymov S.S. ${ }^{2}$ \\ ${ }^{1}$ Tomsk Polytechnic University, Tomsk, Russia, lysenkoen@tpu.ru \\ 2 E.A. Buketov Karaganda University, Karaganda, Kazakhstan
}

\begin{abstract}
The paper analyzes the possibility of describing the accelerated sintering of lithium-titanium compacts under high-power radiation effects in terms of classical physical models developed by Kuczynski and Johnson based on the kinetic analysis of ferrite compact densification. LiTi ferrites synthesized by ceramic technology in laboratory conditions were investigated. The first portion of the compacts was sintered in air in thermal ovens at $900-1100^{\circ} \mathrm{C}$ for 30 minutes at a heating rate of $900^{\circ} \mathrm{C} / \mathrm{min}$. The second portion of the compacts was sintered in a similar mode but using a radiation-thermal method: the exposure to pulsed electron beam with energy of 1.5-2.0 MeV using the ILU-6 accelerator. The geometrical dimensions of the compacts were measured before and after sintering to determine shrinkage. Mathematical approximation of the experimental shrinkage data showed the discrepancy of both models to describe sintering of LiTi ferrite samples both under thermal sintering conditions and radiationthermal effect.
\end{abstract}

Keywords: lithium ferrite, radiation-thermal sintering, shrinkage, kinetic analysis

\section{Introduction}

Diffusion-controlled solid-phase reactions form the basis of many physicochemical processes [1-4]. At the same time, interactions in the solid phase are among the slowest reactions, since the limiting reaction stage in most cases is related to the diffusive mass transfer of components through the layer of reaction products, and to the crossing of the activation barriers that separate the phases of heterogeneous systems [57]. Classical methods used to accelerate solid-phase reactions associated with increased temperature are naturally limited due to the thermal stability of materials [8-11]. Therefore, the development of methods to further increase the efficiency of solid-phase reactions is inevitably associated with unconventional methods used to supply energy to the reacting systems. The most promising method of stimulating solid-phase processes is the effect of high-current fluxes of ionizing radiation [12-22]. The advantages of this method are the selectivity of local overheating of phase boundaries, inertia-free heating, the ionizing effect and increased diffusion permeability of the medium due to defect formation.

In radiation materials science, accelerated electron fluxes are most widely used variety of ionizing radiation [23, 24]. This is due firstly to the progress in accelerator technology and relatively small, but powerful electron accelerators. Secondly, electron beams exhibit convenient geometrical parameters, easy to control irradiation mode, and do not induce radioactivity, they are cheaper compared to other sources of ionizing radiation, and highly efficient in conversion of electricity into electron beam energy [25-28].

The first studies that addressed solid-phase transformations in inorganic materials performed using high-temperature electron irradiation, which featured higher reaction rates, improved product quality, and reduced temperature of the processes, were investigations oxide under these specific conditions conducted for the synthesis of europium [29] and lithium ferrite compacts [30]. In [31], the authors report data on the effect of the high-power electron beam on solid-state inorganic synthesis occurring in a number of systems. It is shown that the magnitude of the effect depends on the following factors: the nature of the system, temperature and dose rate. Similar effects were found during the synthesis of a number of complex oxides in [32]. These effects were also used in current studies in sintering of MnZn ferrite [33, 34] and radiationthermal synthesis of lithium-substituted ferrites [35-37].

During sintering of powder compacts of oxide systems (including ferrite ones), the radiation effect is characterized by increased speed of compact densification, especially at initial stages of sintering. However, 
despite a large number of studies, the mechanism of the radiation intensification of sintering processes remains unclear. There are dispersed opinions on the mechanisms of the radiation activity of the processes: from overheating of grain boundaries and grain surfaces, which provides inter particle slip, to diffusion processes enhanced by the generation of non-equilibrium vacancies.

From the above, it can be seen that there are no objective assumptions to choose an adequate model of radiation sintering. The efficiency of radiation used to control solid-phase reactions depends crucially on the depth of understanding of the mechanism of its effect on the nature of the processes. The most common point of view on the mechanism of radiation acceleration of solid phase sintering implies a radiation-stimulated increase in the mobility of diffusing particles. However, a priori use of these ideas to interpret the effects of radiation-thermal sintering is inappropriate, since the diffusion mechanisms of mass transfer are not the only ones occurring during compact sintering. Therefore, this study analyzes the possibility of describing accelerated sintering of lithium-titanium compacts under powerful radiation effects by classical physical models based on the kinetic analysis of ferrite compact densification.

\section{Experiment}

The study explores mainly lithium-titanium ferrite powders synthesized from a mechanical mixture of oxides and carbonates containing (weight \%): $\mathrm{Li}_{2} \mathrm{CO}_{3}-11.2 ; \mathrm{TiO}_{2}-18.65 ; \mathrm{ZnO}-7.6 ; \mathrm{MnCO}_{3}-2.74$; the rest is $\mathrm{Fe}_{2} \mathrm{O}_{3}$. The components are weighed as specified and then ground and mixed for 1 hour using a vibration grinding mill with distilled water previously added to the mixture in a mixture-water ratio (by weight) equal to $1: 2$. After treatment in the vibration grinding mill, the mixture is dried at $80{ }^{\circ} \mathrm{C}$ at normal atmospheric pressure for 24 hours and then rubbed through a 0.7 mesh sieve. Distilled water is added to the resulting powder in the amount of $10 \%$ of the powder weight, and then it is briquetted. The briquettes are heated in thermal furnaces at a rate of $200^{\circ} \mathrm{C} / \mathrm{h}$ up to $900^{\circ} \mathrm{C}$, held at this temperature for 6 hours and cooled to room temperature, after which they are crushed, ground and sieved through a 0.9 mesh sieve.

After re-grinding in the vibration grinding mill for 45 minutes, $\mathrm{Bi}_{2} \mathrm{O}_{3}$ dissolved in concentrated nitric acid in suspension $(0.22$ weight $\%)$ is added to the powder and mixed in the ball mill for 4 hours. The quality of the powder is determined using the method developed by us. To prepare the powder compact, $10 \%$ solution of polyvinyl alcohol is added to the synthesized mixture in the amount of 12 weight $\%$ of the mixture, and the resulting mixture is alternately rubbed through 0.7 and 0.45 mesh sieves. To study the sintering processes, the compact samples are made by cold single-action compaction in the form of pellets (15 mm in diameter and $2 \mathrm{~mm}$ thick). The compaction pressure is typically chosen experimentally for each specific ferrite composition according to the determined dependence of the volume density $(\rho)$ of the samples on the compaction pressure $(P)$ (Fig. 1).

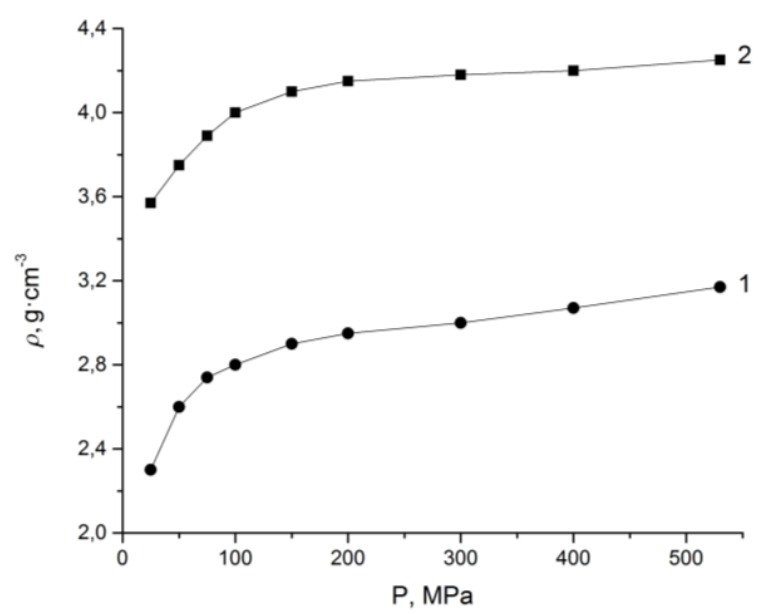

Fig.1. Dependence of the density of ferrite compacts on compaction pressure: 1) raw samples; 2) sintered samples

The compaction pressure varies within 25-500 MPa. The compacts are sintered in the thermal furnace at $900-1100^{\circ} \mathrm{C}$ for 30 minutes in air at a heating rate of $900^{\circ} \mathrm{C} / \mathrm{min}$. High speed is achieved by placing the compact samples into the furnace heated to a predetermined point. As follows from Fig. 1, the dependence of the density of the sintered samples on compaction pressure is similar to the dependence of densification of 
raw samples (curves 1,2). It is established that at pressures below $40 \mathrm{MPa}$ the compacts exhibit low density, and at pressures above $250 \mathrm{MPa}$, the samples have cracks and delamination (over-compaction phenomenon).

Thus, the most optimal compaction pressure to provide an acceptable density of both raw and sintered samples is in the range of 110-200 MPa. The study employed the following pressing mode: $P=130 \mathrm{MPa}$, the holding time of the material under pressure is $1 \mathrm{~min}$; and two modes of compact sintering: radiationthermal $(R T)$ and thermal $(T)$ modes. $R T$-sintering was carried out by irradiation of the compacts by the pulsed electron beam with energy of $1.5-2.0 \mathrm{MeV}$ at the ILU-6 accelerator developed at the Institute of Nuclear Physics (SB, Novosibirsk, Russia) [38]. The beam current in pulse was 0.5-0.9 A, the duration of irradiation pulse was $500 \mu \mathrm{s}$, the pulse repetition rate was $5-50 \mathrm{~Hz}$, and the heating rate of the compacts was $1000^{\circ} \mathrm{C} / \mathrm{min}$. The samples were irradiated in a lightweight fireclay box with a bottom $15 \mathrm{~mm}$ thick. The side of the box exposed to irradiation was covered with a radiation-transparent protector with a mass thickness of $0.1 \mathrm{~g} / \mathrm{cm}$. The temperature was measured using a check sample placed in close proximity to the compacts sintered.

$T$-sintering was carried out in a preheated chamber electric furnace, which provided a heating rate comparable to the rate of radiation heating. The sintering cell design and temperature control technique are similar to those used in $R T$-sintering. Both sintering modes were carried out in the air. To date, a large number of sintering theories have been advanced at the atomic level. We used the model representations by Kuczynski [39] and Johnson [40] to perform the analysis. Kuczynski's theory in its simplest and clearest form contains fundamental principles underlying most of the sintering models. Johnson's theory takes into account a number of additional significant factors, such as coexistence of several types of mass transfer (volume and grain boundary diffusion, transfer through the gas phase) and change in the geometric dimension of grains during sintering.

\section{Results and Discussion}

\subsection{Kuczynski's model}

According to this model, the interpenetration of powder grains (the initial phase of densification) is carried out through the mass transfer by the mechanism of volume $(V)$ or grain boundary $(G B)$ diffusion [2427]. In volume diffusion, the densification kinetics is described by the equation:

$$
\left(\frac{\Delta L}{L}\right)_{V}=\frac{\left(31 \gamma V D_{V} / R T\right)^{2 / 5} \tau^{2 / 5}}{4 a^{6 / 5}} \tau^{2 / 5} .
$$

According to the mechanism of grain boundary diffusion, the kinetics takes the form:

$$
\left(\frac{\Delta L}{L}\right)_{G B}=\frac{\left(96 \gamma \delta V D_{G B} / R T\right)^{1 / 3} \tau^{1 / 3}}{4 a^{4 / 3}} \tau^{1 / 3} .
$$

In (1) and (2): $\Delta L / L$ is linear shrinkage; $a$ is the radius of spherical grains; $\gamma$ is the coefficient of surface tension; $V$ is the material molar volume; $\delta$ is the grain boundary width; $D_{\mathrm{V}}$ and $D_{\mathrm{GB}}$ are coefficients of volume and grain boundary diffusion, respectively; $T$ is sintering temperature; $\tau$ is sintering time. Mathematical approximations in (1) and (2) limit the applicability of these expressions to $\Delta L / L \approx 6 \%$.

The experimental results were processed as follows. The diameters of the sintered samples were measured, and the kinetic dependences of diameters in the time interval of 0-400 s were constructed for all sintering modes, in which the diameter linear shrinkage did not exceed $10 \%$. After compaction, the diameters of all samples were similar and equaled $15.05 \mathrm{~mm}$. This value $d_{0}$ was taken as $L_{0}$. The intermediate values of diameters with respect to the diameters measured were taken from the constructed dependences $d=f(\tau)$ to calculate linear shrinkage $\Delta L / L=\Delta d / d$.

Figures 2 and 3 show dependences plotted in double logarithmic coordinates: $\Delta d / d=f(\tau)$ for thermal (line 1) and radiation-thermal (line 2) sintering modes. The dependencies are straight lines. This indicates the exponential functional dependence of shrinkage on sintering time [39]. Using the least squares method, the densification dependences were approximated by exponential functions:

$$
\frac{\Delta d}{d_{0}}=A \tau^{n},
$$

where $A$ is the temperature dependent constant; $n$ is the sintering mode dependent exponent. 


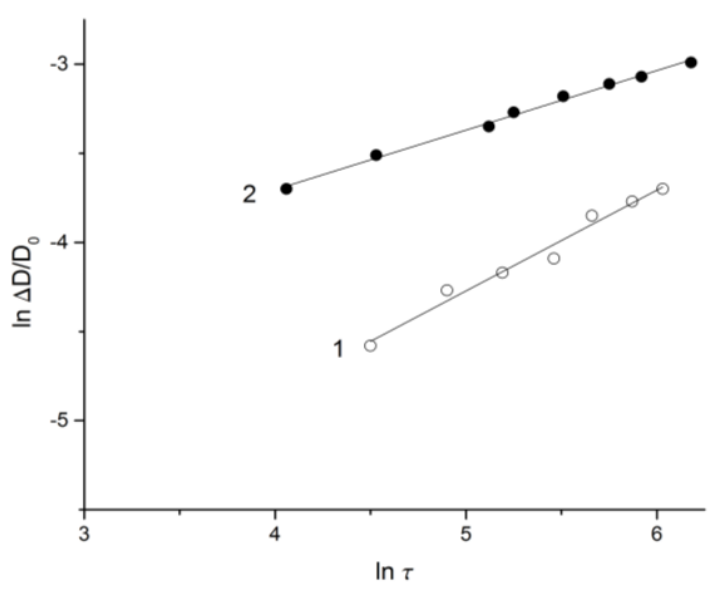

Fig.2. Dependence of linear shrinkage on time of sintering at $900{ }^{\circ} \mathrm{C}$ : 1) $T$-sintering; 2) $R T$-sintering.

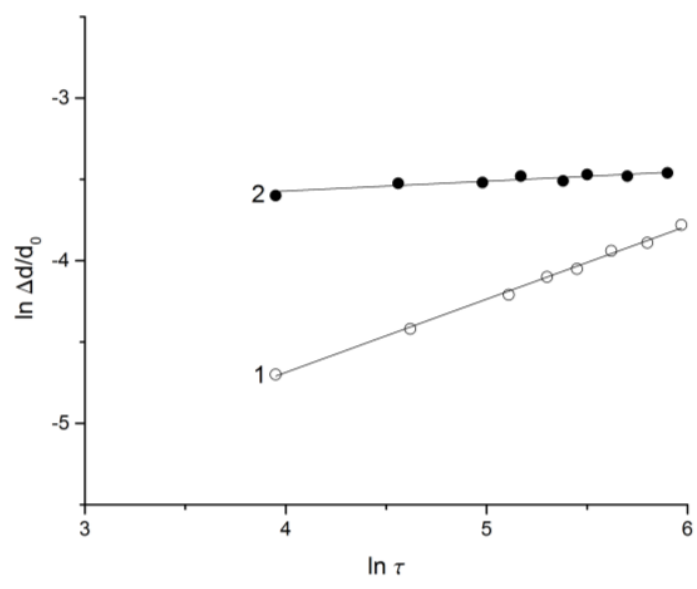

Fig.3. Dependence of linear shrinkage on time of sintering at $1000{ }^{\circ} \mathrm{C}$ : 1) $T$-sintering; 2) $R T$-sintering.

Table 1 presents the numerical values of the exponential function parameters for both sintering modes in the temperature range of $900-1100^{\circ} \mathrm{C}$.As can be seen, the Kuczynski's model does not satisfy the experimental results despite the exponential dependence of the initial densification. This follows from the fact that the exponent, which characterizes the mechanism of mass transfer to the contact area, monotonously changes with temperature. This may be due to a continuous change in the mechanism of mass transfer (when the temperature changes), which contradicts the theory of Kuczynski.

Table 1 - Parameters of exponential functions (3)

\begin{tabular}{|c|c|c|c|c|}
\hline \multirow{2}{*}{ Sintering temperature, ${ }^{\circ} \mathrm{C}$} & \multicolumn{4}{|c|}{ Sintering mode } \\
\cline { 2 - 5 } & $n$ & $A$ & $n$ & $A$ \\
\cline { 2 - 5 } & $n$ & $5.4 \cdot 10^{-5}$ & 0.38 & $5.7 \cdot 10^{-3}$ \\
\hline 900 & 1.11 & $1.4 \cdot 10^{-3}$ & 0.31 & $1.1 \cdot 10^{-2}$ \\
\hline 950 & 0.61 & $3.4 \cdot 10^{-3}$ & 0.18 & $3.5 \cdot 10^{-2}$ \\
\hline 1000 & 0.51 & $1.8 \cdot 10^{-2}$ & 0.14 & $4.7 \cdot 10^{-2}$ \\
\hline 1050 & 0.28 & $0.7 \cdot 10^{-2}$ & 0.09 & $6.7 \cdot 10^{-2}$ \\
\hline 1100 & 0.46 & \multicolumn{3}{|c|}{ Thermal } \\
\hline
\end{tabular}

It should be noted that the correspondence of the parameter $n$ to any sintering mechanism depends on the contact geometry. However, this correspondence should not depend on temperature. In this regard, we can conclude that the mechanism of sintering LiTi ferrites does not satisfy the Kuczynski's model.

\subsection{Johnson's model}

Johnson's model assumes that mass transfer, which leads to material densification, is performed through diffusion in the volume and along the grain boundary. For the case of sintering the compact from spheres of similar diameter, in which the number of contacts per sphere does not depend on time, the relationship between the linear shrinkage rate $V=\frac{d}{d_{0}}(\Delta L / L)$ and the current contact geometry is expressed by equation [40]:

$$
\frac{X^{3} R}{X+R} V=\frac{2 \gamma \Omega D_{V} S}{\pi k T a^{3} X}+\frac{4 \gamma \Omega D_{G B}}{k T a^{4}},
$$

where $X=x / a$ is relative neck radius; $\Omega$ is atomic volume; $R$ is theminimum radius of the neck surface curvature divided by the sphere radius; $S$ is the neck surface area divided by $a^{2}$; x is the inner radius of the spherical contact neck; a is the radius of spherical grains. Knowing $\Delta d / d$ and $X$, the values of $R$ and $S$ can be calculated by the equations:

$$
R=\frac{(\Delta d / d)^{2}-2(\Delta d / d)+X^{2}}{2(1-X)},
$$




$$
S=4 \pi R[Q(1+R) \cos Q-R \sin Q],
$$

where $Q=\sin ^{-1}[(1-(\Delta d / d)) /(1+R)]$.

When graphically depicting the left side of equation (4) depending on $S / X$, the coefficients of volume diffusion and diffusion along the grain boundaries can be calculated from the slope of curves and their intersections.

Equation (4) can be greatly simplified if we confine to the analysis of the initial stages of isothermal densification. In this case, the relative neck radius $X=x / a$ can be expressed analytically through linear shrinkage $(\Delta d / d)$ :

$$
\frac{\Delta d}{d}=\left(\frac{x}{2 S}\right)^{2}, X=\frac{x}{a}=2 \sqrt{\Delta d / d} .
$$

Thus, to apply equation (4) with the approximation (5), it is necessary to know the kinetic dependences of shrinkage $y=f(\tau)$ of the average grain size $\bar{a}=f(\tau)$ only. The approximation limits the range of changes $\Delta d / d$ to $10 \%$. Microstructural analysis of ferrite samples sintered in thermal and radiation modes at $1100^{\circ} \mathrm{C}$ was performed to determine the kinetic changes in the grain size. The measurement results are presented in Figure 4. It can be seen that recrystallization growth of grains becomes evident at $\tau>1800 \mathrm{~s}$ in both sintering modes. That is, at the initial stages of isothermal sintering, changes in grain sizes can be neglected and $a=$ const in (4). The processing of experimental data according to equation (4) was carried out as follows. The kinetic dependencies of the initial densification were approximated by exponential functions (3) with the parameters given in Table 1. The shrinkage derivatives for the sintering time range of $0-400 \mathrm{~s}$ (60 s steps) were calculated by differentiation (3). Then, according to (5), $X$ was calculated and the values of $S, R, Q$ were determined for each point of time.

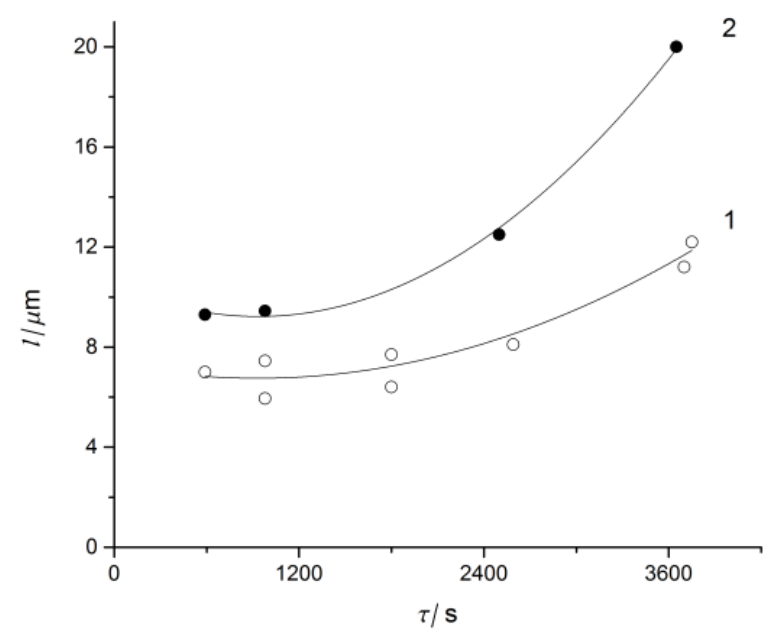

Fig.4. Dependence of the average grain size on time of sintering at $1100{ }^{\circ} \mathrm{C}$ : 1) $T$-sintering; 2) $R T$-sintering.

The function graphs were plotted taking $\operatorname{TRX}^{3} \frac{d(\Delta d / d)}{d \tau} /(X+R)$ in (4) as a function and $S / X$ as an argument. Some graphs are presented in Figures 5 and 6 . According to (4), in case the Johnson's model is valid, the curves should be obtained in given coordinates, the slope of which is proportional to the volume diffusion coefficient $D_{\mathrm{V}}$, and the intercept in the ordinate axis is proportional to the boundary diffusion coefficient $D_{\mathrm{GB}}$.

However, the experimental curves behave arbitrarily depending on the sintering mode and temperature. In particular, the curves obtained show either non-linearity (Fig. 5, curve 1 and Fig. 6, curve 2) or negative values of the intercepts in the ordinate axis (Fig. 5, curve 1 and Fig. 6, curves 1,2). Nonlinearity of the curves contradicts equation (4). Negative values of the slope and intercepts indicate negative diffusion coefficients, which has no physical sense.

Thus, the analysis of the experimental results within the Johnson's model shows inefficiency of this model for describing the sintering process of LiTi ferrite samples under both thermal sintering and radiationthermal exposure. 


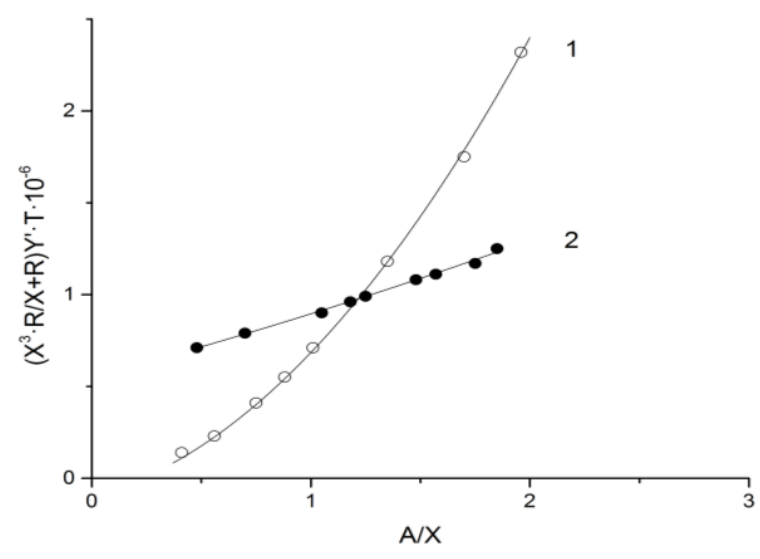

Fig.5. Dependence of densification on geometric characteristics of the grains at $900{ }^{\circ} \mathrm{C}$ :

1) $T$-sintering; 2) $R T$-sintering.

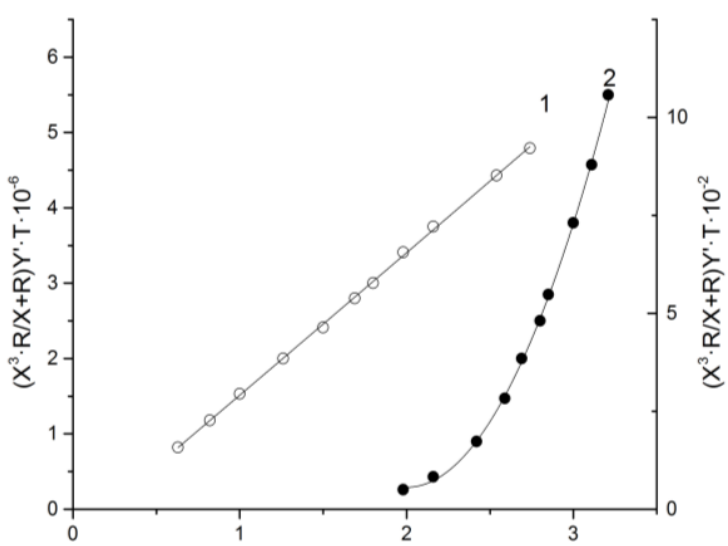

Fig.6. Dependence of densification on geometric characteristics of the grains at $1000{ }^{\circ} \mathrm{C}$ :

1) $T$-sintering; 2) $R T$-sintering.

\section{Conclusion}

Classical physical models cannot be apparently used to describe the kinetics of ferrite compact densification due to the infeasibility of simplifying assumption (simple geometric shapes of powder grains, equal grain size, etc.). In this regard, the current stage of development of physics of sintering does not allow the description of the processes of electron-beam sintering of ferrite ceramics using physical models, and the phenomenological approach is preferable. This approach, in particular, will allow aquantitative comparison of radiation and thermal sintering modes to identify their features and to interpret these in terms of some hypotheses.

\section{Acknowledgements}

This research was supported by the Ministry of Education and Science of the Russian Federation in part of the "Science" program. The experiments on equipment and participation in the scientific conference were funded from Tomsk Polytechnic University Competitiveness Enhancement Program grant.

\section{REFERENCES}

1 Surzhikov A.P., Pritulov A.M., Lysenko E.N., Sokolovskii A.N.,Vlasov V.A., Vasendina E.A. Influence of solid-phase ferritization method on phase composition of lithium-zinc ferrites with various concentration of zinc. Journal of Thermal Analysis and Calorimetry. 2012, Vol. 109, pp. 63 - 67. https://doi.org/10.1007/s10973-011-1366-3.

2 Surzhikov A.P., Lysenko E.N., Malyshev A.V., Pritulov A.M., Kazakovskaya O.G. Influence of mechanical activation of initial reagents on synthesis of lithium ferrite. Russian Physics Journal. 2012, Vol. 55, №.6, pp. 672 - 677. https://doi.org/10.1007/s11182-012-9865-7.

3 Sharma P., Uniyal P. Investigating thermal and kinetic parameters of lithium titanate formation by solid-state method. Journal of Thermal Analysis and Calorimetry. 2017, Vol.128, pp. 875 - 882. https://doi.org/10.1007/s10973-0165977-6.

4 Sun Yu, Wan Zhipeng, Hu Lianxi, Wu Binghua, Deng Taiqing. Characterization on Solid Phase Diffusion Reaction Behavior and Diffusion Reaction Kinetic of Ti/Al. Rare Metal Materials and Engineering.2017,Vol. 46, pp. 2080 - 2086. https://doi.org/10.1016/S1875-5372(17)30183-2.

5 Durgadsimi S.U., Chougule S.S., Kharabe R.G., Mathad S.N., Rendale M.K. Solid-state synthesis and structural features of $\mathrm{Li}_{0.5} \mathrm{Ni}_{0.75-x / 2} \mathrm{Fe}_{2} \mathrm{O}_{4}$ ferrites. International Journal of Self-Propagating High-temperature Synthesis. 2019, Vol. 28, pp. 71-73. https://doi.org/10.3103/S1061386219010060.

6 Cook W., Manley M. Raman characterization of $\square$ - and $\square-\mathrm{LiFe}_{5} \mathrm{O}_{8}$ prepared through a solid-state reaction pathway. Journal of Solid State Chemistry. 2010, Vol.183, pp. $322 \square$ [326. doi.org/10.1016/j.jssc.2009.11.011.

7 Berbenni V., Marini A., Matteazzi P., et al. Solid-state formation of lithium ferrites from mechanically activated $\mathrm{Li}_{2} \mathrm{CO}_{3}-\mathrm{Fe}_{2} \mathrm{O}_{3}$ mixtures. Journal of the European Ceramic Society. 2003, Vol.23, pp. 527 - 536. https://doi.org/10.1016/S0955-2219(02)00150-4.

8 Rakshit S.K., Parida S.C., Naik Y.P., Venugopal V. Thermodynamic studies on lithium ferrites. Journal of Solid State Chemistry. 2011,Vol.184, pp. 1186 - 1194. https://doi.org/10.1016/j.jssc.2011.03.033. 
9 El-Shobaky G.A., Ibrahim A.A. Solid-solid interactions between ferric oxide and lithium carbonate and the thermal stability of the lithium ferrites produced. Thermochimica Acta. 1987,Vol.118, pp. 151-158. https://doi.org/10.1016/0040-6031(87)80079-5.

10 Senyut V.T. Study of synergistic effect of mechanical activation and high pressure and high temperature sintering on the structure of the material based on boron nitride. Eurasian Physical Technical Journal. 2020, Vol.17, pp. 19-25. https://doi.org/10.31489/2020NO1/19-25.

11 Gundlach E.M., Gallagher P.K. Thermogravimetric determination of the oxygen non-stoichiometry in $\mathrm{Ni}_{0.563} \mathrm{Zn}_{0.188} \mathrm{Fe}_{2.25} \mathrm{O}_{4+}$ and $\mathrm{Ni}_{0.375} \mathrm{Zn}_{0.375} \mathrm{Fe}_{2.25} \quad \mathrm{O}_{4+}$. Thermochimica Acta. 1998, Vol.318, pp. $15 \quad-\quad 20$. https://doi.org/10.1016/S0040-6031(98)00324-4.

12 Ancharova U.V., Mikhailenko M.A., Tolochko B.P., et al. Synthesis and Staging of the Phase Formation for Strontium Ferrites in Thermal and Radiation Thermal Reactions. IOP Conference Series: Materials Science and Engineering. 2015, Vol. 81, Article number 012122. https://doi.org/10.1088/1757-899X/81/1/012122.

13 Zhuravlev V.A., Naiden E.P., Minin R.V., et al. Radiation-thermal synthesis of W-type hexaferrites. IOP onference Series: Materials Science and Engineering. 2015, Vol. 81, 012003. https://doi.org/10.1088/1757899X/81/1/012003.

14 Oane M., Toader D., Iacob N., Ticos C.M. Thermal phenomena induced in a small tungsten sample during irradiation with a few MeV electron beam: Experiment versus simulations. Nuclear Instruments and Methods in Physics Research Section B: Beam Interactions with Materials and Atoms. 2014, Vol. 337, pp. 17 - 20. https://doi.org/10.1016/j.nimb.2014.07.012.

15 Karipbayev Zh., Alpyssova G., Mussakhanov D., Lisitsyn V., Kukenova A., Tulegenova A. Time-resolved luminescence excited with $\mathrm{N}_{2}$ laser of YAG: CE Ceramics formed by electron beam assisted synthesis. Eurasian Physical Technical Journal. 2020, Vol.17, pp. 73 - 76. https://doi.org/10.31489/2020NO1/73-76.

16 Vasoya N.H., Vanpariya L.H., Sakariya P.N., Timbadiya M.D., Pathak T.K., Lakhani V.K., Modi K.B. Synthesis of nanostructured material by mechanical milling and study on structural property modifications in $\mathrm{Ni}_{0.5} \mathrm{Zn}_{0.5} \mathrm{Fe}_{2} \mathrm{O}_{4}$. Ceram. Int. 2010, Vol.36, pp. 947 - 954. https://doi.org/10.1016/j.ceramint.2009.10.024.

17 Jiang W., Wang L., Wang X. Studies on surface topography and mechanical properties of TiN coating irradiated by high current pulsed electron beam, Nucl. Instr. Meth. Phys. Res.Sect. B 2018, Vol.436, pp. 63 - 67.

18 Kavanlooee M., Hashemi B., Maleki-Ghaleh H., Kavanlooee J. Effect of annealing on phase evolution, microstructure, and magnetic properties of nanocrystalline ball-milled LiZnTi ferrite, J. Electronic materials. 2012 , Vol.41, pp. 3082 - 3086. https://doi.org/10.1007/s11664-012-2235-y

19 Naiden E.P., Minin R.V., Itin V.I., Zhuravlev V.A. Influence of Radiation-Thermal Treatment on the Phase Composition and Structural Parameters of the SHS Product Based on W-Type Hexaferrite, Russ. Phys. J. 2013 , Vol. 56, pp. 674 - 680.https://doi.org/10.1007/s11182-013-0084-7

20 Šepelák V., Wibmann S., Becker K.D. Magnetism of nanostructured mechanically activated and mechanosynthesized spinel ferrites, J. Magn. Magn. Mat. 1999, Vol. 203, pp. 135 - 137. https://doi.org/10.1016/S03048853(99)00248-6

21 Ivanov A.S., Maznev V.P., Ovchinnikov V.P., Svinin M.P., TolstunN.G. Present State of Works on Development of Electron Accelerators for Energy Consuming Processes at Efremov Research Institute. Prospects and Challenges in Application of Radiation for Treating Exhaust Gases, Warsaw, 2007.

22 Shirvanedeh H.I., Douk H.S., Farhood B. Improving and optimizing the structure of a cylindrical ionization chamber for radiation protection dosimetry: Guard electrode and chamber wall. Radiation Physics and Chemistry, 2019, Vol.163, pp. 45 - 51. https://doi.org/10.1016/j.radphyschem.2019.04.058

23 Matsuyama S., Ishii K., Fujisawa M., et al. Upgrading of the 4.5 MV Dynamitron accelerator at Tohoku University for microbeam and nanobeam applications. Nucl. Instr. Meth. B. 2009, Vol. 267, pp. 2060 - 2064. https://doi.org/10.1016/j.nimb.2009.03.028.

24 Lisitsyn V.,Lisitsyna L.,DauletbekovaA., et al. Luminescence of the tungsten-activated $\mathrm{MgF}_{2}$ ceramics synthesized under the electron beam. Nucl. Instr. Meth. B. 2018, Vol.435, pp. $263 \quad-\quad 267$. doi.org/10.1016/j.nimb.2017.11.012.

25 Salimov R.A., Cherepkov V.G., Golubenko J.I., et al. High power electron accelerators of ELV-series: status, development, applications. Radiation Physics and Chemistry. 2000, Vol. 57, pp. $661 \quad-\quad 665$. https://doi.org/10.1016/S0969-806X(99)00486-7.

26 Cleland M.R., Parks L.A. Medium and high-energy electron beam radiation processing equipment for commercial applications. Nuclear Instruments and Methods in Physics Research Section B: Beam Interactions with Materials and Atoms. 2003, Vol. 208, pp. 74 - 89. https://doi.org/10.1016/S0168-583X(03)00672-4.

27 Mehnert R. Review of industrial applications of electron accelerators. Nuclear Instruments and Methods in Physics Research Section B: Beam Interactions with Materials and Atoms. 1996, Vol. 113, pp. 81 - 87. https://doi.org/10.1016/0168-583X(95)01344-X.

28 Martins M.N., Silva T.F. Electron accelerators: History, applications, and perspectives. Radiation Physics and Chemistry. 2014, Vol. 95, pp. 78 - 85. https://doi.org/10.1016/j.radphyschem.2012.12.008. 
29 Neronov V.A., Voronin A.P., Tatarintseva M.I., et al. Sintering under a high-power electron beam. Journal of the Less Common Metals. 1986, Vol. 117, pp. 391 - 394. https://doi.org/10.1016/0022-5088(86)90065-2.

30 Surzhikov A.P., Lysenko E.N., Malyshev A.V., et al. Study of the Radio-Wave Absorbing Properties of a Lithium-Zinc Ferrite Based Composite. Russian Physics Journal. 2014, Vol. 57, №. 5, pp. $621 \square \square 626$. https://doi.org/10.1007/s11182-014-0284-9.

31 Boldyrev V.V., Voronin A.P., Gribkov O.S., et al. Radiation-thermal synthesis current achievement and outlook. Solid State Ionics. 1989, Vol. 36, pp.1 - 6. https://doi.org/10.1016/0167-2738(89)90051-9.

32 Lyakhov N.Z., Boldyrev V.V., Voronin A.P., et al. Electron beam stimulated chemical reaction in solids. Journal of Thermal Analysis. 1995. Vol.43, pp.21 - 31. https://doi.org/10.1007/BF02635965.

33 Kostishin V.G., Andreev V.G., Korovushkin V.V., et al. Preparation of $2000 \mathrm{NN}$ ferrite ceramics by a complete and a short radiation-enhanced thermal sintering process. Inorganic Materials. 2014, Vol.50, pp. 317 - 323. https://doi.org/10.1134/S0020168514110089.

34 Kostishyn V.G., Komlev A.S., Korobeynikov M.V., et al. Effect of a temperature mode of radiation-thermal sintering the structure and magnetic properties of Mn-Zn-ferrites. Journal of Nano- and Electronic Physics. 2015, Vol.7, 04044.

35 Lysenko E.N., Surzhikov A.P., Vlasov V.A., et al. Synthesis of substituted lithium ferrites under the pulsed and continuous electron beam heating. Nuclear Instruments and Methods in Physics Research Section B: Beam Interactions with Materials and Atoms. 2017, Vol. 392, pp.1 - 7. https://doi.org/10.1016/j.nimb.2016.11.042.

36 Surzhikov A.P., Malyshev A.V., Lysenko E.N., et al. Structural, electromagnetic, and dielectric properties of lithium-zinc ferrite ceramics sintered by pulsed electron beam heating. Ceramics International. 2017, Vol. 43, pp. 9778 - 9782. https://doi.org/10.1016/j.ceramint.2017.04.155.

37 Surzhikov A.P., Pritulov A.M., Lysenko E.N., et al. Dependence of lithium-zinc ferrospinel phase composition on the duration of synthesis in an accelerated electron beam. Journal of Thermal Analysis and Calorimetry. 2012, Vol. 110, pp. 733 - 738. https://doi.org/10.1007/s10973-011-1947-1.

38 Auslender V.L. ILU-type electron accelerator for industrial technologies. Nuclear Instruments and Methods in Physical research B. 1994, Vol. 89, pp. 46 - 48. https://doi.org/10.1016/0168-583X(94)95143-8.

39 Kuczynski G.C. Model experiments and the theory of sintering. Sintering Key Papers, Elsevier Applied Science, London and New York. 1990, pp.501 - 502.

40 Johnson D.L. A general model for the intermediate stage of sintering. Journal of the American Ceramic Society. 1970, Vol. 53, pp. 574 - 579.https://doi.org/10.1111/j.1151-2916.1970.tb15969.x. 